\title{
A double-blind, randomized, placebo- controlled trial of combined calcitriol and ergocalciferol versus ergocalciferol alone in chronic kidney disease with proteinuria
}

Paweena Susantitaphong ${ }^{*}$, Siriwan Nakwan, Sadudee Peerapornratana, Khajohn Tiranathanagul, Pisut Katavetin, Nattachai Srisawat, Kearkiat Praditpornsilpa and Somchai Eiam-Ong

\begin{abstract}
Background: $\mathrm{KDOQ}$ guideline suggests that nutritional vitamin D should be supplemented in chronic kidney disease (CKD) patients who have vitamin D insufficiency/deficiency. However, there are scarce data regarding the additional benefit of active vitamin D supplement in CKD patients who were receiving nutritional vitamin D supplement. This study was conducted to explore the effect of adding active vitamin $D$ to nutritional vitamin $D$ supplement on proteinuria and kidney function in CKD with vitamin D insufficiency/deficiency.

Methods: This double-blind, randomized placebo-controlled trial was performed to answer the above question. Sixty-eight patients with CKD stage 3-4, urine protein to creatinine ratio (UPCR) $>1 \mathrm{~g} / \mathrm{g}$, and serum $25 \mathrm{OH}-\mathrm{D}$ level $<30 \mathrm{ng} / \mathrm{mL}$ were enrolled. Patients were randomly assigned to receive 12-week treatment with oral ergocalciferol plus placebo $(n=36)$ or oral ergocalciferol plus calcitriol $(n=32)$.

Results: The mean baseline values of UPCR of both groups were comparable $(3.6 \pm 3.8 \mathrm{~g} / \mathrm{g}$ in combined group and $3.5 \pm 3.0 \mathrm{~g} / \mathrm{g}$ in ergocalciferol group). Following 12-week treatment, there were significant reductions in UPCR from baseline in both groups $(2.3 \pm 2.1 \mathrm{~g} / \mathrm{g}$ in combined group and $2.4 \pm 2.0 \mathrm{~g} / \mathrm{g}$ in ergocalciferol group). The percentage reductions in UPCR of both groups were not significantly different. The mean eGFR and blood pressure did not differ between baseline and 12-week follow-up and between both groups. No severe hypercalcemia or serious side effects were noted in both groups.
\end{abstract}

Conclusions: The proteinuria lowering effect of ergocalciferol in CKD patients with vitamin D deficiency was demonstrated. Additional calcitriol supplement did not have more effects on proteinuria.

Trial registration: (Thai Clinical Trials Registry (TCTR) 20140929002). Date of registration: September 27, 2014.

Keywords: Vitamin D deficiency, CKD, Proteinuria, Ergocalciferol, Calcitriol

\section{Background}

Chronic kidney disease (CKD) is one of the most extremely important non-communicable diseases that has significant morbidity and mortality consequences [1]. The major complications related to CKD include cardiovascular disease, infectious complications, and mineral and bone disorder (MBD). A recent meta-

\footnotetext{
* Correspondence: pesancerinus@hotmail.com

Division of Nephrology, Department of Medicine, King Chulalongkorn

Memorial Hospital, Faculty of Medicine, Chulalongkorn University, 1873,

Rama 4 Rd., Pathumwan, Bangkok 10330, Thailand
}

analysis demonstrated that lower estimated glomerular filtration rate (eGFR) and higher albuminuria were each independently associated with end-stage renal disease (ESRD) and mortality. Both eGFR and albuminuria were more strongly associated with ESRD than mortality in CKD patients [2].

Vitamin D deficiency/insufficiency is a common problem in CKD patients due to dysregulation of vitamin D metabolism from renal insufficiency [3]. Although the definite critical serum $25(\mathrm{OH}) \mathrm{D}$ level and benefits of $25(\mathrm{OH}) \mathrm{D}$ supplement in CKD patients remain controversial, In the 
2003 Kidney Disease Outcomes Quality Initiative (KDOQI) guideline suggested that patients with serum $25(\mathrm{OH}) \mathrm{D}$ levels $<30 \mathrm{ng} / \mathrm{mL}$ should receive supplementation with nutritional vitamin $\mathrm{D}$. The dosage regimens were recommended to be the same as general population [4].

Besides the classical action of vitamin D as an important regulator of mineral bone metabolism, recent evidence points to other important functions in different target organs including renal, cardiovascular systems, and immune response regulation [5]. Vitamin D deficiency was associated with a higher annual incidence of albuminuria, decreased eGFR, and independently predicted 5-year incidence of albuminuria [6]. Vitamin D deficiency was also a significant risk factor for the development of CKD stage 3-5 [7, 8]. In addition, vitamin D deficiency is independently associated with a higher risk of $50 \%$ increase in baseline serum creatinine, ESRD, or death in patients with type II diabetic nephropathy (DN) [9].

Previous cohort studies showed that daily cholecalciferol, a nutritional vitamin $\mathrm{D}$, supplement had a beneficial effect in decreasing albuminuria in CKD and DN [10, 11]. Active vitamin $\mathrm{D}$ has been demonstrated to lessen renin-angiotensin aldosterone system (RAAS) and intraglomerular pressure, and might ameliorate renal injury by reducing fibrosis, apoptosis, and inflammation in animal models [12]. Several randomized controlled trials in patients with proteinuric kidney disease demonstrated the benefit of calcitriol or paricalcitol supplement in decreasing proteinuria [13-15]. However, there were no randomized controlled trials (RCTs) exploring the effect of the additional benefit of active vitamin D supplement in CKD patients who were receiving nutritional vitamin $\mathrm{D}$ supplement. Therefore, this study was conducted to explore the effect of combined nutritional vitamin $\mathrm{D}$ and active vitamin D supplement on proteinuria and kidney function in CKD with vitamin D insufficiency/deficiency.

\section{Methods}

\section{Study design and participants}

The study was performed during July 2014 and February 2015 in adult Thai CKD patients with proteinuria and vitamin $\mathrm{D}$ insufficiency/deficiency at the outpatient clinic at King Chulalongkorn Memorial Hospital (KCMH), Bangkok, Thailand. The study was approved by the Institutional Review Board of the Faculty of Medicine, Chulalongkorn University (Bangkok, Thailand; IRB.093/57) with clinical trial registration (Thai Clinical Trials Registry (TCTR) 20140929002; date of registration: September 27, 2014). All participants received information of study details before giving written informed consent. The inclusion criteria included eGFR of $15-60 \mathrm{~mL} / \mathrm{min}$ per $1.73 \mathrm{~m}^{2}$, age 18 years or older, proteinuria greater than $1 \mathrm{~g} /$ day, and serum vitamin D (25-OH) level less than $30 \mathrm{ng} / \mathrm{mL}$. The eGFR was calculated with serum creatinine concentrations by using Thai-MDRD formula [16]. Exclusion criteria included active glomerulonephritis, receiving immunosuppressive drugs within 3 months before enrollment, adjusting RAAS blockade such as angiotensin-converting-enzyme inhibitors or angiotensin II receptor blockers (ACEIs/ARBs) within 3 months before enrollment, and serum calcium level more than $10.2 \mathrm{mg} / \mathrm{dL}$.

This study was a double-blind RCT of ergocalciferol, a nutritional vitamin $\mathrm{D}$, with or without calcitriol, an active vitamin $\mathrm{D}$, in $\mathrm{CKD}$ with proteinuria and vitamin $\mathrm{D}$ insufficiency/deficiency. We randomly assigned eligible patients in a 1:1 ratio to 40,000 units/week of ergocalciferol plus identical placebo or 40,000 units/week of ergocalciferol plus calcitriol $(0.5 \mu \mathrm{g}$, two times per week) for 12 weeks.

Patients were examined at baseline, 6-week, and 12-week. Blood pressures, adverse events, concomitant drug treatment, and adherence to drug regimens were also recorded. At baseline, 6-week, and 12-week, the blood chemistry parameters including serum creatinine, cystatin $C$, calcium, phosphate, albumin, intact parathyroid hormone (iPTH) were measured while urine specimen collection at first void urine including urine protein to creatinine ratio $(\mathrm{g} / \mathrm{g})$ were determined. The dosages of ACEIs or ARBs were not allowed to adjust during the follow-up period. Other antihypertensive treatments were initiated or increased in dose for controlling blood pressure following the KDIGO guideline $(<130 / 80 \mathrm{mmHg})$ [17]

\section{Randomization and masking}

Computer-generated concealed randomization schedules, each with permuted block sizes of four, were created. When an eligible patient had been enrolled, the research assistant used sealed opaque envelopes to allocate the patient to the next sequential randomization number. This study was double blinded. Patients were not informed which group they were randomly allocated to. The laboratory personnel processing the samples also had no knowledge of each patient's group assignment. The physicians who took care of the patients were also masked.

\section{Outcomes}

The primary endpoint was the percentage change in urine protein to creatinine ratio (UPCR) which was calculated by using the values obtained at baseline and after 12 weeks of follow-up period. Secondary endpoints were the proportion of patients who achieved at least $30 \%$ reduction in UPCR, the change of kidney functions, blood pressure level, and blood chemistry. The safety endpoints and all adverse events were classified and confirmed by personals who were masked to treatment assignment. 


\section{Statistical analysis}

The aim of this study was to determine the effect of additional calcitriol supplement in CKD patients who were receiving ergocalciferol supplement on proteinuria. As there were no published RCTs, we calculated and found that a sample size of 35 patients per arm was needed for $80 \%$ power to detect a difference in proteinuria of $-1.0 \mathrm{~g} / \mathrm{g}$ [standard deviation (SD) 1.5] between ergocalciferol alone and ergocalciferol plus calcitriol group with two-sided significance level of 0.05 . The expected reduction in a urine proteinuria was based on our own clinical setting. The per protocol analysis was used for all efficacies and safety analyses. Patients who lost follow up and had some missing data for calculating the change values in the efficacy and safety measures between two time points were excluded from the analyses. Continuous variables were reported as mean \pm standard deviation. Categorical variables were reported in terms of frequency and percentage. For normally distributed variable, the differences in changes between the two groups were analyzed by unpaired $t$-test. The differences in changes within groups were analyzed by pair $t$-test. For non-normally distributed variable, Mann-Whitney $U$-test was used to analyze differences between the two groups. Categorical outcomes were analyzed by chi-square or Fisher exact test. The statistical analyses were performed by using the SPSS version 16.0 statistical software program.

\section{Results}

Of 112 eligible patients, 74 were enrolled and randomly assigned to ergocalciferol plus calcitriol (combined group, $n=36$ ) or ergocalciferol plus placebo (ergocalciferol group, $n=38$ ). All randomly assigned patients received their allocated treatment and had at least one post-randomization follow-up visit. Two patients were lost to follow-up in ergocalciferol group and 4 patients in combined group (Fig. 1).

Baseline demographic, clinical and biochemical characteristics, and concomitant treatments were balanced between both groups. The main cause of CKD was DN (57.4\%). The participants randomly allocated to the ergocalciferol group were more likely to have lower serum $25(\mathrm{OH})$ D levels than those in the combined group (Table 1).

\section{Effect on proteinuria}

The mean baseline values of UPCR of both groups were comparable $(3.6 \pm 3.8 \mathrm{~g} / \mathrm{g}$ in combined group and $3.5 \pm$ $3.0 \mathrm{~g} / \mathrm{g}$ in ergocalciferol group). After 12-week treatment, there were significant reductions of UPCR in both groups $(2.3 \pm 2.1 \mathrm{~g} / \mathrm{g}$ in combined group and $2.4 \pm 2.0 \mathrm{~g} / \mathrm{g}$ in ergocalciferol group) (Fig. 2). The percentage reductions in UPCR of both groups were not significantly different $(-25.5 \%, 95 \%$ CI -9.2 to -41.8 in combined group, $-23.7 \%$; $95 \%$ CI -7.0 to -40.5 in ergocalciferol group). More than $50 \%$ of patients had $30 \%$ reduction on proteinuria in both groups (56.3\% in combined group and 52.8\% in ergocalciferol group).

Subgroup analysis by cause of CKD, the level of proteinuria, and status of receiving RAAS blockade were performed. The mean change in UPCR from baseline to the end of treatment in $\mathrm{DN}$ seemed to be greater than non-DN $[-31.3 \%$ vs. $-15.3 \%$ in ergocalciferol group; and $-28.1 \%$ vs $-21.2 \%$ in combined group] (Fig. 3a). In addition, the mean change in UPCR seemed to be more robust in case of nephrotic range proteinuria than non-

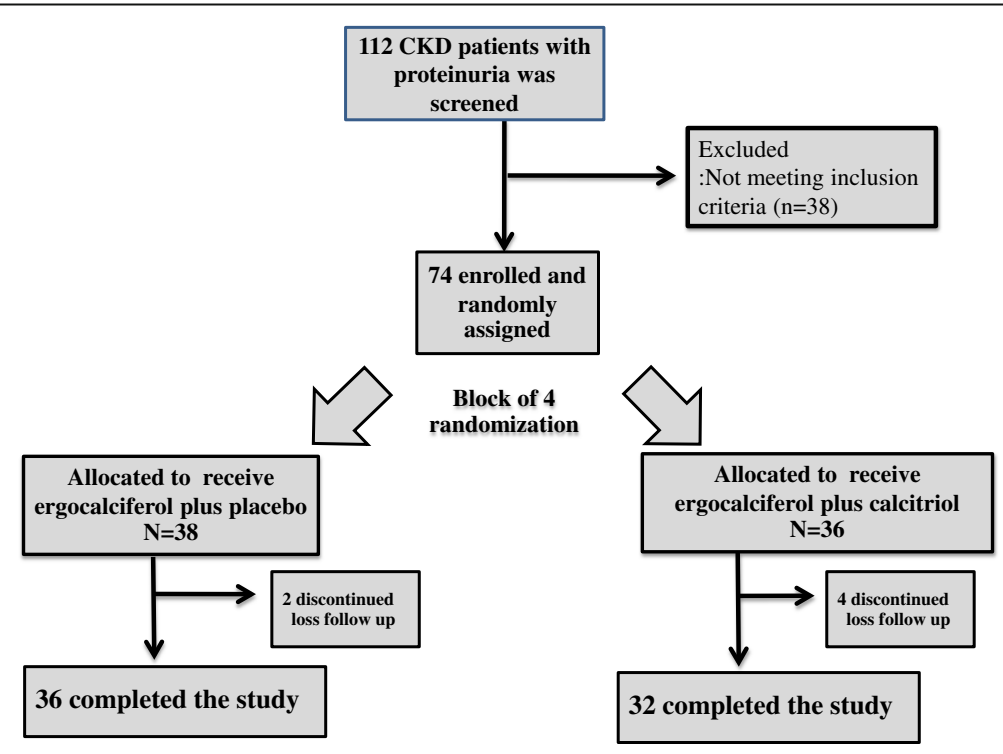

Fig. 1 Flow diagram showing recruitment and follow-up of patients 
Table 1 Baseline characteristics of the study population

\begin{tabular}{|c|c|c|c|}
\hline Demographic & Ergocalciferol plus placebo $(n=36)$ & Ergocalciferol plus calcitriol $(n=32)$ & $P$-value \\
\hline Age (years) & $62.56 \pm 12.91$ & $63.13 \pm 11.29$ & 0.85 \\
\hline Male sex (\%) & $15(41.67)$ & $17(53.13)$ & 0.35 \\
\hline \multicolumn{4}{|l|}{ Cause of CDK } \\
\hline - Diabetic nephropathy & 19 (52.78) & $20(62.5)$ & 0.43 \\
\hline - Chronic glomerulonephritis & $4(11.11)$ & $4(12.5)$ & 0.88 \\
\hline - IgA nephropathy & $1(2.78)$ & $2(6.25)$ & 0.48 \\
\hline - Focal segmental glomerulosclerosis & $3(8.33)$ & $1(3.13)$ & 0.35 \\
\hline - Polycystic kidney disease & $1(11.11)$ & $0(0)$ & 0.31 \\
\hline - Unknown & $8(22.22)$ & $5(15.63)$ & 0.49 \\
\hline \multicolumn{4}{|l|}{ Blood pressure $(\mathrm{mmHg})$} \\
\hline - Systolic & $136.69 \pm 14.56$ & $137.75 \pm 14.78$ & 0.77 \\
\hline - Diastolic & $75.75 \pm 10.46$ & $77.13 \pm 11.68$ & 0.61 \\
\hline Serum creatinine (mg/dL) & $2.19 \pm 0.9$ & $2.43 \pm 0.9$ & 0.29 \\
\hline eGFR (ml/min/1.73 m²) & $39.29 \pm 11.26$ & $37.29 \pm 11.44$ & 0.47 \\
\hline Serum cystatin C (mg/L) & $2.07 \pm 0.61$ & $2.13 \pm 0.67$ & 0.70 \\
\hline 25-hydroxy vitamin D level (ng/ml) & $15.89 \pm 6.59$ & $19.26 \pm 5.12$ & 0.02 \\
\hline \multicolumn{4}{|l|}{ Urine protein to creatinine ratio $(\mathrm{g} / \mathrm{g})$} \\
\hline - mean $\pm S D$ & $3.47 \pm 3.01$ & $3.61 \pm 3.75$ & 0.87 \\
\hline Serum calcium (mg/dl) & $9.4 \pm 0.49$ & $9.27 \pm 0.47$ & 0.27 \\
\hline Serum phosphorus (mg/dl) & $3.58 \pm 0.58$ & $3.8 \pm 0.78$ & 0.17 \\
\hline Serum albumin (g/dl) & $3.83 \pm 0.42$ & $3.95 \pm 0.34$ & 0.21 \\
\hline Serum intact parathyroid hormone $(\mathrm{pg} / \mathrm{mL})$ & $85.11 \pm 47.42$ & $88.57 \pm 64.49$ & 0.60 \\
\hline Serum $\mathrm{HbA1C}(\%)$ & $7.17 \pm 0.78$ & $7.40 \pm 0.59$ & 0.31 \\
\hline \multicolumn{4}{|l|}{ Antihypertensive drug (\%) } \\
\hline$-\mathrm{ARB}$ & $8(22.22)$ & $11(34.38)$ & 0.27 \\
\hline - ACE inhibitor & $10(27.78)$ & $8(25)$ & 0.79 \\
\hline
\end{tabular}

nephrotic range $[-38.7 \%$ vs $-14.2 \%$ in ergocalciferol group; and $-28.7 \%$ vs $-23.8 \%$ in combined group (Fig. 3b). However, the mean changes in UPCR in whom receiving RAAS blockade or non-receiving RAAS blockade were not significantly different. $[-30.6 \%$ vs $-16.9 \%$ in ergocalciferol group; and $-17.2 \%$ vs $-36.2 \%$ in combined group] (Fig. 3c).

\section{Effect on kidney function}

eGFR determined by serum creatinine (Thai MDRD) and by cystatin $\mathrm{C}$ (CKD-EPI) did not change during the study period from baseline to the end of treatment and there were no significant differences between the ergocalciferol group and the combined group (Fig. 4).

\section{Effect on blood pressure}

There were no significant changes in mean systolic and diastolic blood pressure values throughout the course of study in either ergocalciferol group or combined group. Mean blood pressure values at baseline were $136.7 \pm$
$14.6 / 75.8 \pm 10.5 \mathrm{mmHg}$ in the ergocalciferol group and $137.8 \pm 14.8 / 77.1 \pm 11.7 \mathrm{mmHg}$ in the combined group (NS). At the end of treatment, mean blood pressure values were $135.6 \pm 17.4 / 77.9 \pm 11.2 \mathrm{mmHg}$ in the ergocalciferol group and 135.8 $\pm 20.0 / 77.9 \pm 11.9 \mathrm{mmHg}$ in the combined group.

\section{Effect on metabolic bone disease}

There were no significant changes of serum calcium, serum phosphate from baseline to the end of treatment as well as between both groups $[9.4 \pm 0.5$ to $9.3 \pm$ $0.6 \mathrm{mg} / \mathrm{dL}$ and $3.6 \pm 0.6$ to $3.8 \pm 0.7 \mathrm{mg} / \mathrm{dL}$ in ergocalciferol group, $9.3 \pm 0.5$ to $9.2 \pm 0.5 \mathrm{mg} / \mathrm{dL}$ and $3.8 \pm 0.8$ to $3.8 \pm 0.6 \mathrm{mg} / \mathrm{dL}$ in combined group]. No severe hypercalcemia, hyperphosphatemia, or serious side effects were noted in both groups (NS).

There were no significant differences between iPTH levels measured at baseline between two groups. Serum iPTH was slightly increased from $85.1 \pm 47.4 \mathrm{pg} / \mathrm{mL}$ at baseline to $86.0 \pm 46.0 \mathrm{pg} / \mathrm{mL}(P=0.83)$ at the end of 


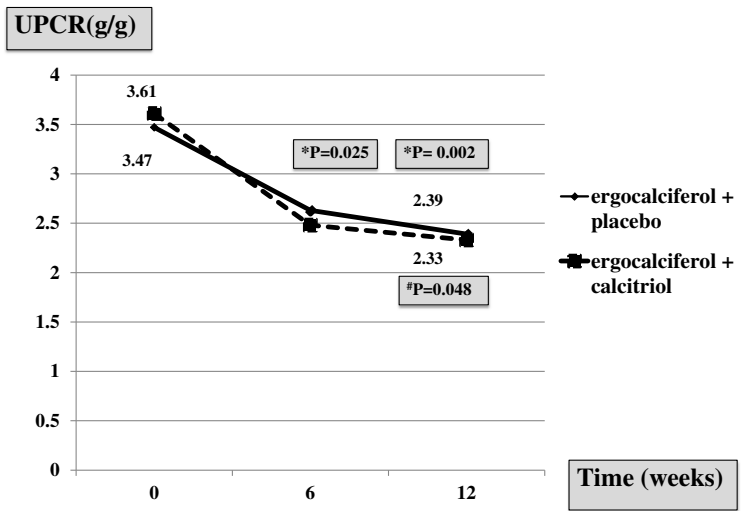

Fig. 2 The absolute net change of UPCR from baseline to 12 weeks follow-up between both groups * $P$-value when compared with baseline in ergocalciferol group " $P$-value when compared with baseline in combined group

treatment in the ergocalciferol group and was significantly decreased from $88.6 \pm 64.5$ to $73.9 \pm 73.3 \mathrm{pg} / \mathrm{mL}$ $(P=0.04)$ in the combined group. Serum iPTH levels in the combined group were significantly lower than the ergocalciferol group at the end of treatment $(P=0.03)$.

Vitamin D levels $(25(\mathrm{OH}) \mathrm{D})$ were significantly increased from baseline to the end of treatment in both ergocalciferol group $(15.9 \pm 6.6$ to $30.6 \pm 11.9 \mathrm{ng} / \mathrm{mL})$ and combined group $(19.5 \pm 5.2$ to $33.4 \pm 11.3 \mathrm{ng} / \mathrm{mL})$, but no statistical significance was observed between both groups.

\section{Discussion}

This study was the first double-blind RCT to explore the additional benefit of calcitriol supplement in CKD patients who were receiving ergocalciferol supplementation on proteinuria and kidney function by using serum cystatin C in CKD patients with vitamin D insufficiency/ deficiency. The study demonstrated that the 12-week course of ergocalciferol supplementation in patients with stage 3-4 CKD and nutritional vitamin D insufficiency/ deficiency could improve proteinuria as well as restored 25 -vitamin D concentrations. Nevertheless, adding calcitriol to ergocalciferal treatment did not provide further beneficial effect for proteinuria (Fig. 2). Moreover, the kidney function monitored by serum creatinine-based and cystatin C-based eGFR did not significantly change from the baseline to the end of treatment in both groups and there were no significant differences between the two groups (Fig. 4). No severe hypercalcemia or serious side effects were noted in both ergocalciferol and combined groups. In addition, blood pressure and glycemic control were not significantly different between two groups.

Although several observational studies in CKD patients have shown the benefit of nutritional vitamin D supplementation in decreasing proteinuria [10, 11], there were no RCTs exploring the benefit of nutritional vitamin $\mathrm{D}$ on proteinuria and renal functions. This RCT demonstrates the benefit of nutritional vitamin $\mathrm{D}$ supplement in proteinuric CKD with vitamin $\mathrm{D}$ insufficiency/deficiency on proteinuria. The potential mechanisms remain to be further explored. One possible mechanism is that normal ranges of 25-(OH) D level achieved by ergocalciferol supplement might be changed to the active form and could activate VDR.

Several previous RCTs of proteinuric kidney disease demonstrated the beneficial effect of calcitriol or paricalcitol supplement on decreasing proteinuria [13-15]. However, there is some inconsistency of the evidence. In RCT of selective vitamin D receptor activation with paricalcitol for reduction of albuminuria in patients with type 2 diabetes (VITAL study), paricalcitol could not demonstrate an additional effect in decreasing albuminuria in DN patients [18]. The different dosage regimens and the levels of proteinuria might affect the results. Indeed, in a recent systematic review and meta-analysis, active vitamin $\mathrm{D}$, vitamin $\mathrm{D}$ compounds, and nutritional vitamin $\mathrm{D}$ showed antiproteinuric effect in CKD patients $[19,20]$. However, these are no available data regarding the role of combined nutritional vitamin $\mathrm{D}$ and active vitamin $\mathrm{D}$ on proteinuria and renal function in CKD patients with vitamin $\mathrm{D}$ insufficiency/deficiency. In the present study, the combined ergocalciferol and calcitriol also resulted in decreasing proteinuria from baseline while the additional effect of combined nutritional and active vitamin $\mathrm{D}$ could not be demonstrated (Fig. 2). Admittedly, the long-term benefit of combined ergocalciferol and calcitriol was not explored in this study.

As such, the present study demonstrates that whenever nutritional vitamin $\mathrm{D}$ is supplemented until achieving the target level, the agent can decrease proteinuria. Vitamin $\mathrm{D}$ and its analogues appear to have similar antiproteinuric effects through activation of the VDR by several mechanisms. Active vitamin D acts as a strong negative endocrine regulator of the RAAS and functions mainly to suppress renin production in experimental studies [21, 22]. Inactive vitamin D and its metabolites (such as $25(\mathrm{OH}) \mathrm{D}$ and $24,25(\mathrm{OH}) 2 \mathrm{D})$ might also have additional effect on proximal tubular cells. 25(OH) D bound to vitamin $\mathrm{D}$ binding protein is endocytosed by megalin-cubilin in the apical membrane of proximal tubular cells. Then, intracellular $25(\mathrm{OH}) \mathrm{D}$ is metabolized to $1,25(\mathrm{OH}) 2 \mathrm{D}$ (calcitriol) by 1- $\alpha$-hydroxylase in the tubular cell [23]. Furthermore, vitamin D has been shown to have anti-fibrotic and immunomodulatory effects by attenuating fibrotic and inflammatory markers in animal and clinical studies [24-26]. The potential mechanism should be explored in further studies. 


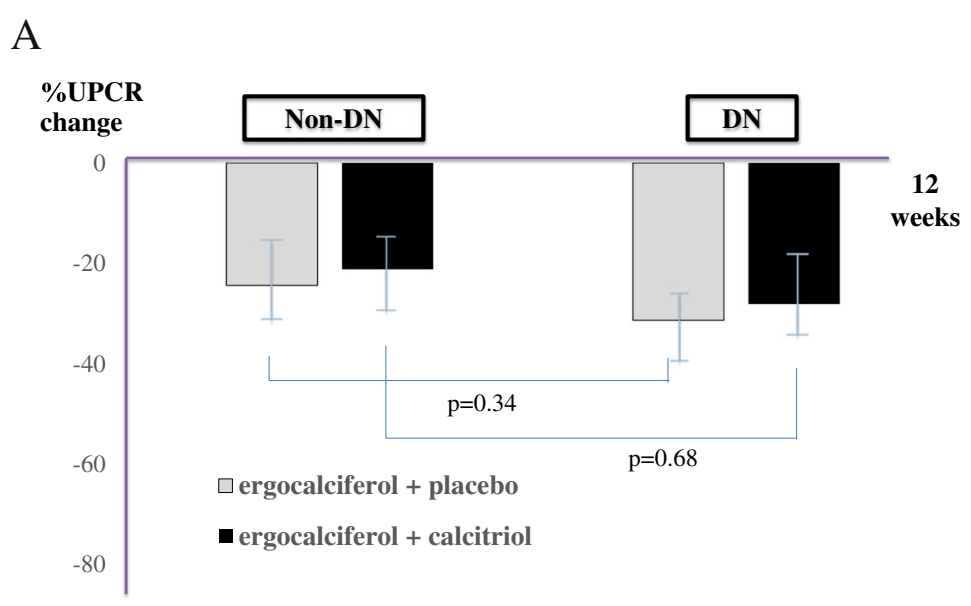

B

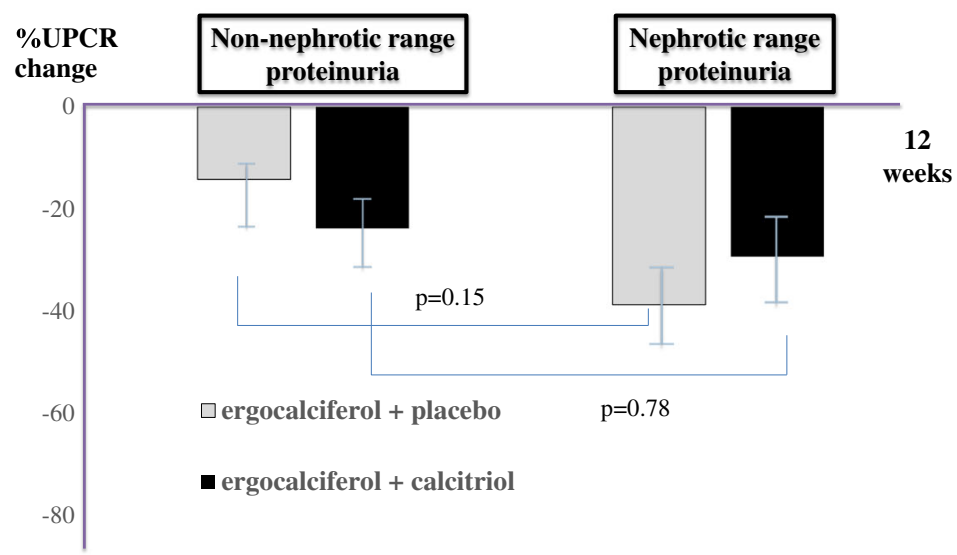

$\mathrm{C}$

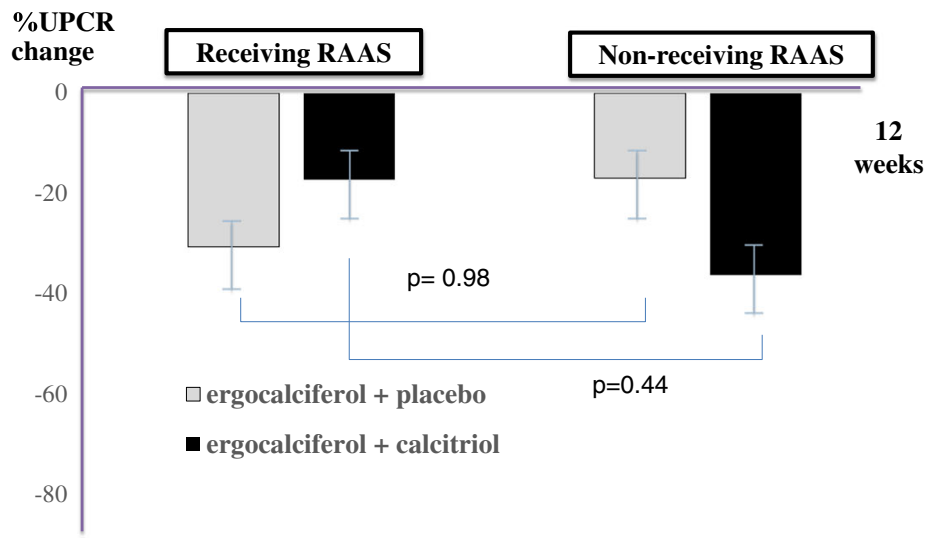

Fig. 3 The percentage change of UPCR from baseline to 12 weeks follow-up between both groups. Subgroup analyses by cause of CKD (3A), Level of proteinuria (3B), Receiving RAAS (3C)

In term of kidney function, vitamin D compounds could not demonstrate the benefit on renal function in CKD patients in a recent systematic review and meta-analysis $[19,20]$. However, the renal function in all included studies was determined by serum creatinine-based formula which might be interfered by increasing muscle mass from vitamin D supplement. New biomarkers such as serum cystatin $C$ should be used for more accurate renal function measurement. Although the serum cystatin $\mathrm{C}$ was used for calculating eGFR in this study (Fig. 4), the benefits of kidney function after vitamin $\mathrm{D}$ supplement were still not proven. A longer study might be required to examine the renal function retardation effect of ergocalciferol with/without calcitriol treatment. 


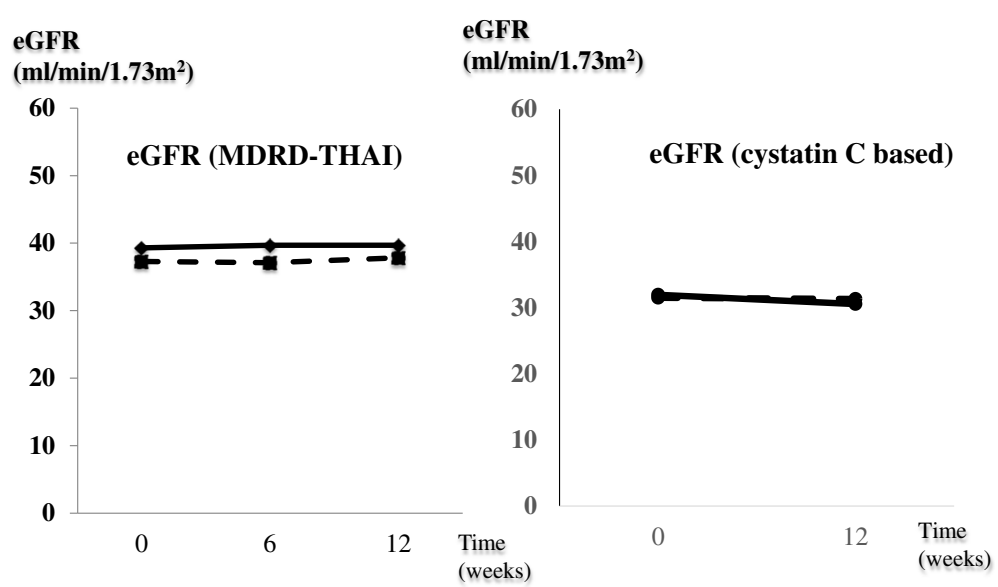

Fig. 4 The absolute net change of eGFR (MDRD-THAI) and eGFR (CKD-epi cystatin c based) from baseline to 12 weeks follow-up between both groups

Regarding metabolic bone disease, although serum calcium and phosphate levels did not change during the treatment in both groups, the benefit of controlling serum iPTH was demonstrated only in the calcitriol group. Therefore, active vitamin $\mathrm{D}$ compound should be used in CKD if the goal is to treat secondary hyperparathyroidism. There are some limitations of the present analysis including small sample size that might result from unexpected high standard deviation, the method of urine protein measurement, office blood pressure monitoring, and short-term follow up.

Finally, according to the $2003 \mathrm{KDOQI}$ guideline, it remains controversial regarding the definite critical serum $25(\mathrm{OH}) \mathrm{D}$ level and benefits of $25(\mathrm{OH}) \mathrm{D}$ supplements in CKD patients [3]. The guideline only mentioned that whenever serum $25(\mathrm{OH})$ D levels $<30 \mathrm{ng} / \mathrm{mL}$, supplementation with nutritional vitamin $\mathrm{D}$ should be initiated using dosing regimens recommended for the general population. The first line use of ergocalciferol or cholecalciferol was recommended due to less hypercalcemia and hyperphosphatemia [4]. In another guideline, the Kidney Disease: Improving Global Outcomes (KDIGO) guideline [27] also provided opinion-based recommendations regarding measurement of $25(\mathrm{OH}) \mathrm{D}$ in patients with CKD. KDIGO recommended correcting 25(OH) D deficiency or insufficiency using treatment strategies used for the general population. Although ergocalciferolprescribing strategy in hemodialysis patients with vitamin D insufficiency/deficiency using the KDOQI and KDIGO guidelines is inadequate to achieve repletion or maintenance of normal vitamin D levels [28], the ergocalciferol dosing in this study can improve and maintain vitamin $\mathrm{D}$ status in CKD patients.

\section{Conclusions}

The effect of ergocalciferol to lower proteinuria in CKD patients with vitamin $\mathrm{D}$ deficiency was demonstrated in this RCT. However, additional calcitriol did not have more proteinuria-lowering effects. Therefore, ergocalciferol should be added for more decreasing proteinuria in CKD patients with proteinuria that cannot tolerate or increase the dosage of RAAS blockade due to any side effects. However, there are some limitations of the present analysis including small sample size and short-term follow up that might result in non-significance on renal function.

\section{Abbreviations}

25-OH-D: 25-hydroxy vitamin D; ACEl: Angiotensin-converting-enzyme inhibitor; ARBs: Angiotensin II receptor blockers; CKD: Chronic kidney disease; CKD-EPI: The Chronic Kidney Disease Epidemiology Collaboration; DN: Diabetic nephropathy; eGFR: Estimated glomerular filtration rate; ESRD: End-stage renal disease; iPTH: Intact parathyroid hormone; KCMH: King Chulalongkorn Memorial Hospital; KDIGO: Kidney disease improving global outcomes; KDOQI: Kidney Disease Outcomes Quality Initiative; MBD: Mineral and bone disorder; MDRD: The Modification of Diet in Renal Disease; RAAS: Renin angiotensin aldosterone system; RCT: Randomized controlled trial; SD: Standard deviation; TCTR: Thai Clinical Trials Registry; UPCR: Urine protein to creatinine ratio; VDR: Vitamin D receptor

\section{Acknowledgement}

The authors would like to thank all the study patients for their participating in this study. This work was supported by Ratchadapiseksompotch Research Fund, Department of Medicine, Faculty of Medicine, Chulalongkorn University, and Thailand research fund Career Development Grant \#RSA5880067. Thailand. Ergocalciferol, calcitriol, and identical placebo were used in this study were granted from Kaspa Pharmaceutical (Thailand) company.

Availability of data and materials

All data generated or analyzed during this study are included in this published article. The datasets analyzed during the current study are available from the corresponding author on reasonable request.

\section{Authors' contributions}

Conception and design: PS, SN, PK. Analysis and interpretation of the data: PS, SN, PK, KP, SE. Drafting of the article: PS, SN, PK, KP, SE. Critical revision of the article for important intellectual content: PS, PK, KP, SE. Final approval of the article: PS, SN, SP, KT, PK, NS, KP, SE. Provision of study materials or patients: PS, SN, SP, KT, PK, NS, KP, SE. Statistical expertise: PS, PK. Administrative, technical, or logistic support: PS, PK, NS, KP, SE. All authors read and approved the final manuscript.

Competing interests

The authors declare that they have no competing interests. 


\section{Consent for publication}

Not applicable.

\section{Ethics approval and consent to participate}

The study was approved by the Institutional Review Board of the Faculty of Medicine, Chulalongkorn University (Bangkok, Thailand; IRB.093/57). Each patient gave his/her consent to participate in the study.

Received: 20 November 2015 Accepted: 4 January 2017

\section{Published online: 14 January 2017}

\section{References}

1. Stanifer JW, Muiru A, Jafar TH, Patel UD. Chronic kidney disease in low- and middle-income countries. Nephrol Dial Transplant. 2016;31(6):868-74. doi:10. 1093/ndt/gfv466. Epub 2016 Feb 4.

2. Astor BC, Matsushita K, Gansevoort RT, van der Velde M, Woodward M, Levey AS, et al. Lower estimated glomerular filtration rate and higher albuminuria are associated with mortality and end-stage renal disease. A collaborative meta-analysis of kidney disease population cohorts. Kidney Int. 2011:79:1331-40.

3. Levin A, Bakris GL, Molitch M, Smulders M, Tian J, Williams LA, et al. Prevalence of abnormal serum vitamin D, PTH, calcium, and phosphorus in patients with chronic kidney disease: results of the study to evaluate early kidney disease. Kidney Int. 2007;71:31-8.

4. National Kidney Foundation. K/DOQI clinical practice guidelines for bone metabolism and disease in chronic kidney disease. Am J Kidney Dis. 2003; 42:S1-201.

5. Susantitaphong P, Eiam-Ong S. Role of Vitamin D in Chronic Kidney Disease. Asian Biomed. (in press).

6. Damasiewicz MJ, Magliano DJ, Daly RM, Gagnon C, Lu ZX, Sikaris KA, et al. Serum 25-hydroxyvitamin D deficiency and the 5-year incidence of CKD. Am J Kidney Dis. 2013;62:58-66.

7. Satirapoj B, Limwannata P, Chaiprasert A, Supasyndh O, Choovichian P. Vitamin $D$ insufficiency and deficiency with stages of chronic kidney disease in an Asian population. BMC Nephrol. 2013;14:206.

8. Izumaru K, Ninomiya T, Nagata M, Usui T, Yoshida D, Yonemoto K, et al. Serum 1,25-dihydroxyvitamin $\mathrm{d}$ and the development of kidney dysfunction in a Japanese community. Circ J. 2014;78:732-7.

9. Fernandez-Juarez G, Luno J, Barrio V, de Vinuesa SG, Praga M, Goicoechea $\mathrm{M}$, et al. $25(\mathrm{OH})$ vitamin $\mathrm{D}$ levels and renal disease progression in patients with type 2 diabetic nephropathy and blockade of the renin-angiotensin system. Clin J Am SocNephrol. 2013;8:1870-6.

10. Molina P, Gorriz JL, Molina MD, Peris A, Beltran S, Kanter J, et al. The effect of cholecalciferol for lowering albuminuria in chronic kidney disease: a prospective controlled study. Nephrol Dial Transplant. 2014;29:97-109.

11. Kim MJ, Frankel AH, Donaldson M, Darch SJ, Pusey CD, Hill PD, et al. Ora cholecalciferol decreases albuminuria and urinary TGF- $\beta 1$ in patients with type 2 diabetic nephropathy on established renin-angiotensin-aldosterone system inhibition. Kidney Int. 2011;80(8):851-60.

12. Kim CS, Kim SW. Vitamin D and chronic kidney disease. Korean J Intern Med. 2014;29:416-27.

13. Krairittichai U, Mahannopkul R, Bunnag S. An open-label, randomized controlled study of oral calcitriol for the treatment of proteinuria in patients with diabetic kidney disease. J Med Assoc Thai. 2012;95 Suppl 3:S41-7.

14. Szeto CC, Chow KM, Kwan BC, Chung KY, Leung CB, Li PK. Oral calcitriol for the treatment of persistent proteinuria in immunoglobulin A nephropathy: an uncontrolled trial. Am J Kidney Dis. 2008:51:724-31.

15. Agarwal R, Acharya M, Tian J, Hippensteel RL, Melnick JZ, Qiu P, et al. Antiproteinuric effect of oral paricalcitol in chronic kidney disease. Kidney Int. 2005;68:2823-8.

16. Praditpornsilpa K, Townamchai N, Chaiwatanarat T, Tiranathanagul K, Katawatin $\mathrm{P}$, Susantitaphong $\mathrm{P}$, et al. The need for robust validation for MDRD-based glomerular filtration rate estimation in various CKD populations. Nephrol Dial Transplant. 2011;26:2780-5.

17. Kidney Disease: Improving Global Outcomes (KDIGO) Blood Pressure Work Group. KDIGO clinical practice guideline for the management of blood pressure in chronic kidney disease. Kidney Int Suppl. 2012;2:337-414.

18. de Zeeuw D, Agarwal R, Amdahl M, Audhya P, Coyne D, Garimella T, et al. Selective vitamin $D$ receptor activation with paricalcitol for reduction of albuminuria in patients with type 2 diabetes (VITAL study): a randomised controlled trial. Lancet. 2010;376:1543-51.
19. de Borst MH, Hajhosseiny R, Tamez H, Wenger J, Thadhani R, Goldsmith DJ. Active vitamin $D$ treatment for reduction of residual proteinuria: a systematic review. J Am SocNephrol. 2013;24:1863-71.

20. Xu L, Wan X, Huang Z, Zeng F, Wei G, Fang D, et al. Impact of vitamin D on chronic kidney diseases in non-dialysis patients: a meta-analysis of randomized controlled trials. PLoS One. 2013;8:e61387.

21. Freundlich $M$, Quiroz $Y$, Zhang Z, et al. Suppression of renin-angiotensin gene expression in the kidney by paricalcitol. Kidney Int. 2008;74(11):1394-402.

22. Li YC, Kong J, Wei M, Chen ZF, Liu SQ, Cao LP. 1,25-Dihydroxyvitamin D(3) is a negative endocrine regulator of the renin-angiotensin system. J Clin Invest. 2002;110(2):229-38.

23. Chesney RW. Interactions of vitamin D and the proximal tubule. Pediatr Nephrol. 2016:31(1):7-14.

24. Kim CS, Joo SY, Lee KE, Choi JS, Bae EH, Ma SK, Kim SH, Lee J, Kim SW. Paricalcitol attenuates 4-hydroxy-2-hexenal-induced inflammation and epithelial-mesenchymal transition in human renal proximal tubular epithelial cells. PLoS One. 2013;8(5):e63186. doi:10.1371/journal.pone.0063186.

25. Mathieu C, Adorini L. The coming of age of 1,25- dihydroxy vitamin D(3) analogs as immunomodulatory agents. Trends Mol Med. 2002;8(4):174-9.

26. Schwarz U, Amann K, Orth SR, Simonaviciene A, Wessels S, Ritz E. Effect of $1,25(\mathrm{OH}) 2$ vitamin $\mathrm{D} 3$ on glomerulosclerosis in subtotally nephrectomized rats. Kidney Int. 1998;53(6):1696-705.

27. Kidney Disease: Improving Global Outcomes (KDIGO) CKD-MBD Work Group. KDIGO clinical practice guideline for the diagnosis, evaluation, prevention, and treatment of chronic kidney disease-mineral and bone disorder (CKD-MBD). Kidney Int Suppl. 2009:113:S1-130.

28. Porter A, Gilmartin C, Srisakul U, Arruda J, Akkina S. Prevalence of 25-OH vitamin $D$ deficiency in a population of hemodialysis patients and efficacy of an oral ergocalciferol supplementation regimen. Am J Nephrol. 2013;37: $568-74$.

\section{Submit your next manuscript to BioMed Central and we will help you at every step:}

- We accept pre-submission inquiries

- Our selector tool helps you to find the most relevant journal

- We provide round the clock customer support

- Convenient online submission

- Thorough peer review

- Inclusion in PubMed and all major indexing services

- Maximum visibility for your research

Submit your manuscript at www.biomedcentral.com/submit
) Biomed Central 